\title{
Multi-component adducts of benzoic acid-fluorobenzoic acid combinations
}

Seera Raviteja $^{1}{ }^{\text {, Suryanarayan Cherukuvada }}{ }^{1}$, T. N. Guru Row ${ }^{1}$

${ }^{1}$ Solid State And Structural Chemistry Unit And Indian Institute Of Science, Bengaluru, India E-mail: seeraraviteja1992@gmail.com

Cocrystals and solid solutions are different yet related crystalline multi-component adducts known to modulate the physicochemical properties of various chemical substances, thus finding applications in pharmaceutical and materials science. Solid solutions are more candid to design than cocrystals as it is well-established that a solid solution is formed by substitutional incorporation of a substance in the crystal lattice of another substance based on the isomorphous and/or isostructural relationships. Thus, a solid solution retains homomeric interactions and adopts the crystal structure of one of the parent substances. In contrast, a cocrystal can manifest for a combination wherein heteromeric interactions outweigh homomeric interactions of individual components. Since the heteromeric interactions are structure-directing, a cocrystal has unique crystal packing and can be readily characterized by X-ray diffraction techniques. Cocrystallization behaviour among various benzoic acid-fluorobenzoic acid combinations is explored to evaluate the propensity of preferred adduct in these combinations. It is reported that benzoic acid-4-fluorobenzoic acid combination forms a solid solutions whereas benzoic acid-pentafluorobenzoic acid combination forms a cocrystal. In the background of these extremes, a systematic cocrystallization study has been undertaken to determine the effect of number of fluorine atoms in the metamorphosis of homomeric interactions to heteromeric ones for combinations containing di-, tri- and tetra-fluorobenzoic acids. The gradation from solid solution (BA-monofluoro BA) to cocrystal (BA-pentafluoro BA) with eutectic phases in certain di- and tri- fluorobenzoic acid combinations is discussed. The study involves crystal structure determination, powder diffraction and thermal analysis and thus allows for deriving general guidelines to generate desired crystal forms by fine tuning hetero- and homomeric interactions.

[1] Yamamoto, N., Taga, T. and Machida, K. (1989) Acta Crystallogr. Sect. B: Struct. Sci. 45, 162.

[2] Reddy, L. S., Bhatt, P. M., Banerjee, R., Nangia, A. and Kruger, G. J. (2007) Chem. Asian J. 2, 505.

[3] Cherukuvada, S., Kaur, R. and Guru Row, T.N. (2016) CrystEngComm. 18, 8528-8555.

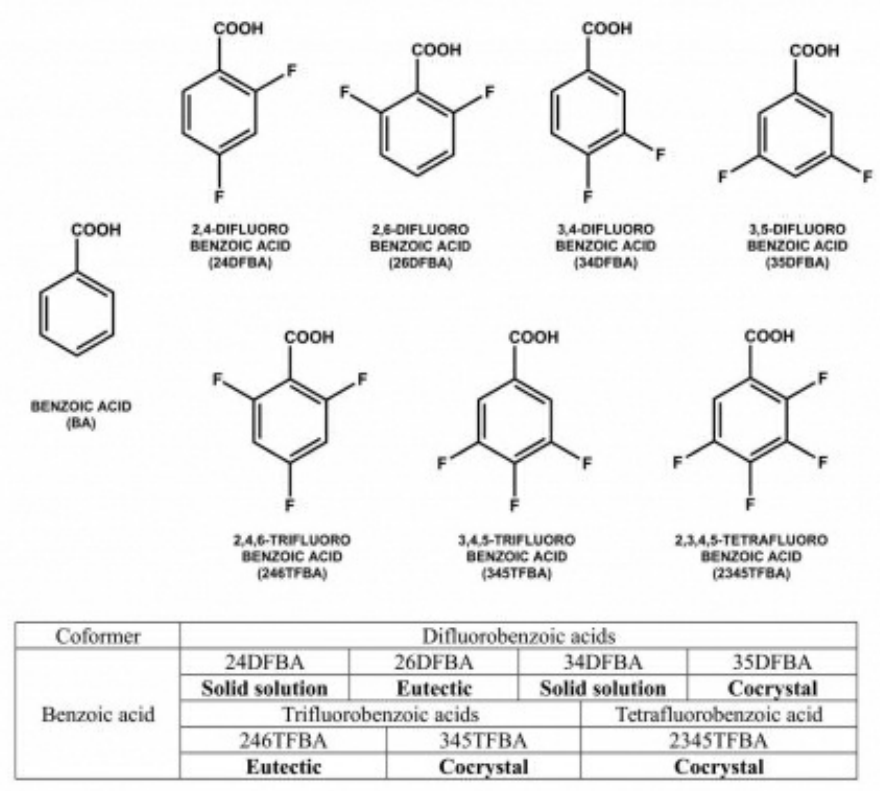

Keywords: Cocrystal, Solid Solution, Eutectic 